\title{
Modificación de la Morfología de una Superficie de Silicio Mediante el Bombardeo con Iones de Oxigeno
}

\author{
Silicon Surfice Morofology Modification thorough Oxigen’s Ions Bombardment \\ A. G. Hernández ${ }^{a}$, Karthik T. V. K. ${ }^{b}$, René Asomoza-Palacio ${ }^{c}$, Yuriy Kudriavtsev ${ }^{d}$
}

\begin{abstract}
:
In this work, the study of nanopatterning formation dynamics of surfaces under ion bombardment was carried out. The bombardment was performed on silicon ( $\mathrm{Si}$ ) surfaces at room temperature by using a positive ion beam of diatomic oxygen $(\mathrm{O} 2+)$. The energy of the ion beam used was $2 \mathrm{keV}$ and the angle of incidence of $45^{\circ}$. The ion dose was varied in the range between $1.25 \times 1018$ ions/cm2 and $7.5 \times 1018$ ions $/ \mathrm{cm} 2$. The results showed that the regime used causes the formation of nanometric structures with a pyramidal shape. The nanopatterns behaves accordingly to the linear models.
\end{abstract}

Keywords:

ion bombardment; nanopatterning; surfaces; oxygen bombardment

\section{Resumen:}

En este trabajo se realizó el estudio de la dinámica de formación de nanopatrones inducida mediante bombardeo iónico. El bombardeo se llevó a cabo sobre superficies de silicio ( $\mathrm{Si}$ ) a temperatura ambiente utilizando un haz de iones positivo de oxigeno diatómico $(\mathrm{O} 2+)$. La energía del haz de iones utilizado fue de $2 \mathrm{keV}$ y el ángulo de incidencia de $45^{\circ}$. La dosis de iones fue variada en el rango de $1.25 \times 1018$ iones $/ \mathrm{cm} 2-7.5$ x1018 iones/cm2. Los resultados muestran que el régimen utilizado provoca la formación de estructuras nanométricas con forma piramidal. El patrón se comporta de acuerdo a los modelos lineales de formación de nano patrones.

Palabras Clave:

bombardeo iónico; formación de nanopatrones; superficies; bombardeo con iones de oxígeno.

\section{1.- INTRODUCCIÓN}

Cuando un haz de partículas se impacta en la superficie de un sólido, una fracción de las partículas incidentes es dispersada y la otra parte se introduce en el interior del sólido provocando una cascada de colisiones [1, 2]. Por lo tanto, la interacción partícula-sólido está gobernada por las propiedades de las colisiones ya que se produce un intercambio de momento entre las partículas y los átomos del sólido [3]. Usualmente se designa a la partícula incidente como "proyectil" y a la materia con la cual interacciona se le denomina "blanco". Las partículas incidentes pueden ser iones o clústeres de átomos. En la cascada de colisiones, todos los átomos se encuentran en movimiento, lo que provoca la retrodispersión de los átomos, ocasionando que aquellos que se encuentran más cercanos a la superficie puedan abandonarla, si reciben una transferencia de energía mayor que la energía de enlace superficial. Si el átomo que sale expulsado del

\footnotetext{
a Autor de Correspondencia: Sección Electrónica del Estado Sólido, Ingeniería Eléctrica, CINVESTAV-IPN, Av. IPN n. 2508, Col. San Pedro Zacatenco, Del. Gustavo A. Madero, Ciudad de México, C.P. 07360, México. Correo electrónico: ghernandez@ cinvestav.mx ${ }^{\mathrm{b}}$ Universidad Autónoma del Estado de Hidalgo, Escuela Superior de Tepeji, Ingeniería Industrial, Av. del Maestro n.41, Col. Noxtongo $2^{\mathrm{a}}$ Sección, Tepeji del Río. Hidalgo C.P. 42855, México.

c Sección Electrónica del Estado Sólido, Ingeniería Eléctrica, CINVESTAV-IPN, Av. IPN n. 2508, Col. San Pedro Zacatenco, Del. Gustavo A. Madero, Ciudad de México, C.P. 07360, México.

d Sección Electrónica del Estado Sólido, Ingeniería Eléctrica, CINVESTAV-IPN, Av. IPN n. 2508, Col. San Pedro Zacatenco, Del. Gustavo A. Madero, Ciudad de México, C.P. 07360, México.
} 
sólido se encuentra eléctricamente cargado, se le conoce como ion secundario.

La expulsión de un ion secundario genera una vacancia en la superficie, una consecuencia evidente de esto es la modificación de la morfología superficial. Sin embargo, resulta interesante que la remoción de material de la superficie bajo determinadas condiciones experimentales, se lleve a cabo de manera simétrica y ordenada dando lugar a la formación de superficies micro y nano-estructuradas con patrones simétricos que se repiten de manera periódica a todo lo largo de la superficie bombardeada [4]. Las morfologías más usuales observadas en superficies bombardeadas se pueden clasificar en cuatro grupos de manera general (a) nano ondas, (b) patrones nanométricos de puntos, (c) patrones piramidales y (d) superficies lisas [5-7].

Los patrones son regulares, simétricos y se extienden sobre toda la superficie irradiada y además poseen aplicaciones tecnológicas importantes tales como [8]:

- Fabricación de materiales optoelectrónicos.

- $\quad$ Almacenamiento magnético.

- Fabricación relativamente sencilla de puntos cuánticos.

- Películas anti reflejantes sobre celdas solares.

- Fabricación de superficies hidrofílicas y/o hidrofóbicas con propiedades antibacteriales

Las características del patrón que se forma en la superficie se encuentran influenciadas por los parámetros experimentales de sputtering, que son:

- $\quad$ Ángulo de incidencia del haz de iones
- $\quad$ Energía de la partícula incidente
- $\quad$ Dosis de ion incidente
- $\quad$ Tipo de blanco
- Temperatura del blanco

En este trabajo se llevó a cabo el estudio del efecto de la dosis de iones en la formación de patrones nanoestructurados provocados por el bombardeo de iones.

\section{2.- DESARROLLO EXPERIMENTAL}

Las muestras de tamaño de $1 \times 1 \mathrm{~cm} 2$ aproximadamente se cortaron de obleas comerciales de silicio (Si). Se realizó una limpieza a temperatura ambiente al sumergir las muestras de manera consecutiva en cada uno de los solventes: acetona, etanol y agua desionizada, utilizando una tina de ultrasonido durante $5 \mathrm{~min}$ (para cada substancia). Finalmente, las muestras fueron enjuagadas en agua desionizada y secadas mediante una pistola de nitrógeno.

Los experimentos se llevaron a cabo en ultra alto vacío (UHV), donde la presión base se encuentra aproximadamente alrededor de 10-11 mbar. El bombardeo fue realizado al estar el substrato a temperatura ambiente utilizando una fuente de iones de $\mathrm{O} 2+$ con energía de $2 \mathrm{keV}$ y corriente de $600 \mathrm{nA}$. El ángulo de incidencia del haz es de $45^{\circ}$ con respecto al plano normal a la superficie.

Después del bombardeo de iones, la topografía de la superficie fue analizada mediante microscopia de fuerza atómica (AFM, por sus siglas en inglés) utilizando un instrumento NTDMT Solver Next. Se utilizó el denominado modo tapping para analizar el relieve de la superficie en 3-D. Todas las mediciones se llevaron a cabo en aire mediante el uso de puntas de silicón con un radio de curvatura de aproximadamente $10 \mathrm{~nm}$. Se tomaron micrografías desde $2 \times 2$ a $50 \times 50$ micras con una densidad de $512 \times 512$ píxeles y una frecuencia de exploración de 1-3Hz. La frecuencia de oscilación del cantiléver fue centrada en $339.39 \mathrm{KHz}$. La rugosidad RMS y las estadísticas de la superficie fueron estudiadas en un área de $5 \times 5 \mu \mathrm{m} 2$ para comparar los resultados.

\section{3.- DISCUSIÓN DE RESULTADOS}

La superficie de Si fue bombardeada con un haz de iones de O2+ cuya energía es de $2 \mathrm{keV}$. Las condiciones experimentales se encuentran resumidas en la tabla 1. La rugosidad de la superficie antes del bombardeo es de $1.42 \mathrm{~nm}$ y este valor incrementa a medida que aumenta la dosis de iones. 
La dosis de iones tiene una relación directamente proporcional con el tiempo de bombardeo. Las muestras fueron bombardeadas desde 5 hasta 30 minutos y los resultados correspondientes se muestran en la Fig. 1.

\begin{tabular}{|c|c|c|c|c|}
\hline Blanco & $\begin{array}{c}\text { Rugosidad } \\
\text { RMS antes } \\
\text { del } \\
\text { bombardeo } \\
\text { [nm] }\end{array}$ & $\begin{array}{c}\text { Rugosidad } \\
\text { RMS } \\
\text { después del } \\
\text { bombardeo } \\
\text { [nm] }\end{array}$ & $\begin{array}{c}\text { Tiempo de } \\
\text { bombardeo } \\
\text { [seg] }\end{array}$ & $\begin{array}{c}\text { Dosis de } \\
\text { iones } \\
\left.\text { [iones } / \mathrm{cm}^{2}\right]\end{array}$ \\
\hline \multirow{5}{*}{$\mathrm{Si}$} & \multirow{5}{*}{1.42} & 24.68 & 300 & $1.25 \mathrm{E}+18$ \\
\hline & & 34.65 & 480 & $2 \mathrm{E}+18$ \\
\hline & & 44.6 & 600 & $2.5 \mathrm{E}+18$ \\
\hline & & 64.19 & 900 & $3.75 \mathrm{E}+18$ \\
\hline & & 91.214 & 1800 & $7.5 \mathrm{E}+18$ \\
\hline
\end{tabular}

bombardeo de iones realizado sobre superficies de silicio.

En la Fig. 1a se muestra la superficie de silicio antes del bombardeo, tal como puede observarse, la superficie es lisa y presenta algunos defectos menores que se pueden atribuir al manejo con pinzas de las muestras, o bien a la oxidación nativa del silicio. Sin embargo, el bombardeo con iones de oxigeno provoca la modificación de la morfología superficial. De la Fig. 1 b a la Fig. 1 f se muestra la evolución de la superficie.

Los resultados nos indican que la incidencia de iones de oxigeno con energía de $2 \mathrm{keV}$ provoca la formación de estructuras piramidales. Al analizar el efecto de la dosis de iones, se observaron las diferentes etapas de la dinámica de formación de los nanopatrones.

La dirección de propagación de las nanoestructuras es paralela a la dirección del haz de iones proyectada sobre la superficie. Así mismo, se observa un engrosamiento de las dimensiones del patrón a medida que incrementa la dosis de iones.

Las primeras etapas de la formación de los nanopatrones se observan en las Figs. 1 b y c, en donde se aprecia que en los primeros instantes del bombardeo se forman sobre la superficie estructuras orientadas. Sin embargo, dichas estructuras no son periódicas y la morfología aún no se encuentra definida.

Durante el bombardeo de iones se presentan mecanismos de rugosidad y de alisamiento que compiten simultáneamente hasta que uno prevalece, dependiendo de las condiciones experimentales. En este caso, los mecanismos de rugosidad dominan dando lugar a la formación de las nanopirámides. Los mecanismos de rugosidad identificados en este experimento son: "Mecanismo de engrosamiento por dispersión" y el de "sombreado" (9).
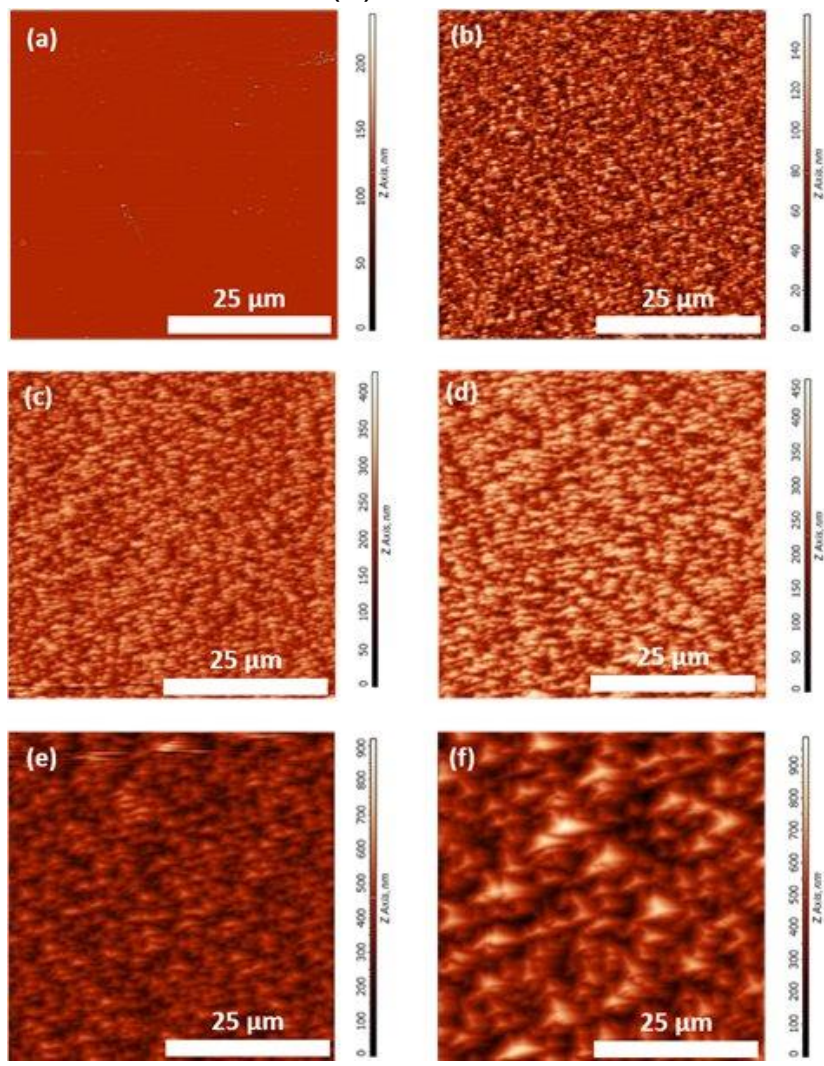

Fig. 1.- Micrografías en $2 D$ obtenidas mediante microscopia de fuerza atómica de las superficies de Si bombardeadas con O2+. La energía del haz es de $2 \mathrm{keV}$ y $45^{\circ}$ de ángulo de incidencia. En (a) se muestra la superficie antes de la incidencia de los iones. La dosis de iones fue variada en cada caso: (b) $1.25 \times 1018$ iones $/ \mathrm{cm} 2$, (c) $2 \times 1018$ iones/cm2, (d) $2.5 \times 1018$ iones $/ \mathrm{cm} 2$, (e) $3.75 \times 1018$ iones/cm2 y (f) $7.5 \times 1018$ iones $/ \mathrm{cm} 2$.

Se identificó que la formación de las nanopirámides toma lugar a partir del bombardeo con una dosis de $3.75 \times 1018$ iones/cm2. A partir de este valor, las dimensiones del patrón sólo 
aumentan de acuerdo con el incremento en la dosis.

En la Fig. 2 se muestra el análisis morfológico de las nanopirámides obtenidas cuando la dosis de iones es $7.5 \times 1018$ iones/cm2. En (a), la flecha verde indica el área donde se realizó el análisis de la sección transversal. En (b) se encuentra el perfil correspondiente. Finalmente, en (c) se despliega la imagen en 3D correspondiente a un área de $5 \times 5$ micras.

La altura promedio de las nanoestructuras es de $600 \mathrm{~nm}$ y el periodo es irregular. En la etapa observada no se alcanza una periodicidad en la distancia a la que están separadas las nanoestructuras. Esto puede ser atribuido a los mecanismos de rugosidad que provocan el engrosamiento de las nanoestructuras.
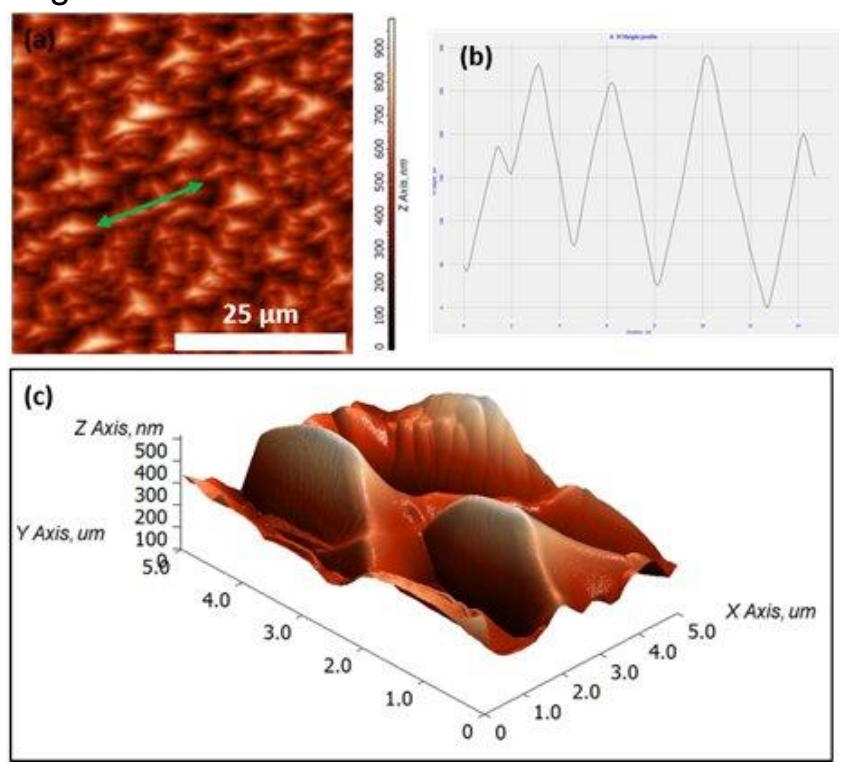

Fig. 2.- Superficie de Si bombardeada con una dosis de iones de $7.5 \times 1018$ iones $/ \mathrm{cm} 2$. (a) imagen 2D de $50 \times 50$ micras. (b) Análisis de la sección transversal de la zona correspondiente a la flecha verde. (c) Representación en 3D de un área de $5 \times 5$ micras de la superficie nanoestructurada.

El mecanismo de formación de las nanopirámides se puede explicar si consideramos el "mecanismo de engrosamiento por dispersión", el cual se ilustra en la Fig. 3. En este proceso, los iones que se reflejan (flechas moradas) contribuyen a la erosión más veloz de una determinada faceta, lo cual a su vez provoca la desaparición de facetas adyacentes de menor tamaño. En la Fig. 3, la superficie representada por una línea continua es bombardeada con iones. En esta superficie, la faceta $l a$ es mucho mayor que la faceta $l b$, entonces hay una mayor cantidad de iones reflejados por la; estos iones impactan en las facetas vecinas por lo que la velocidad de erosión $v a$ es mayor que $v b$, lo que provoca la desaparición de las facetas de menor tamaño. Esto provoca el agrandamiento de los patrones, a medida que aumenta la dosis de iones.

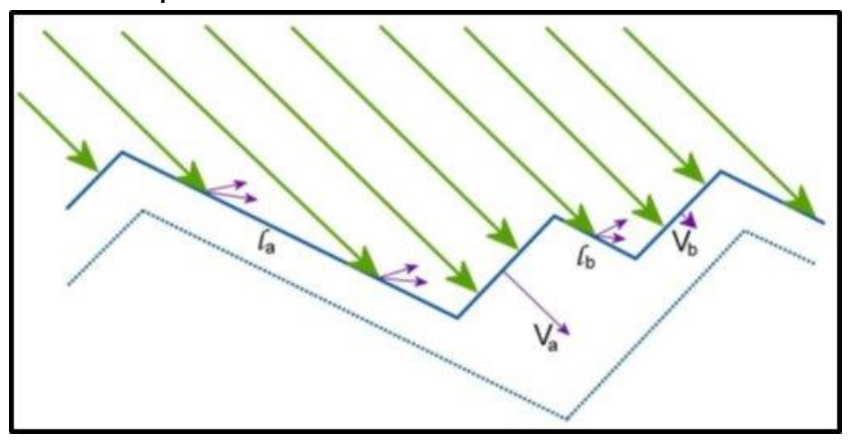

Fig. 3.- Diagrama esquemático del mecanismo de engrosamiento de las facetas de la superficie debido a la reflexión de iones (Modificado de 9).

\section{4.- CONCLUSIONES}

Se realizó el estudio del bombardeo de superficies de silicio a baja energía del haz de iones. Resulta importante llevar a cabo tal estudio debido a que la nueva generación de espectrómetros de masas de iones secundarios trabaja en los regímenes de energía aquí estudiados. Los resultados obtenidos muestran el aumento de la rugosidad superficial, lo que afecta a la medición de los perfiles en profundidad. Por otra parte, la formación de nanopatrones tiene importantes aplicaciones tecnológicas que deben ser ampliamente exploradas.

\section{AGRADECIMIENTOS}

Los autores quisieran agradecer al Consejo Nacional de Ciencia y Tecnología (CONACYT) del gobierno mexicano por aportar los fondos para el desarrollo de este trabajo, en especial al proyecto CB-2016 No. 254903.

\section{Referencias}

[1] E.S. Mashkova, V.A. Molchanov, Medium-energy ion scattering by solid surfaces. Part I, Radiat. Eff. 16 (1972) 143-187. doi:10.1080/00337577208231218. 
[2] E.S. Mashkova, V.A. Molchanov, Medium-energy ion scattering by solid surfaces part II, Radiat. Eff. 23 (1974) 215-270. doi: $10.1080 / 00337577408244188$.

[3] M.V.R. Murty, Sputtering: the material erosion tool, Surf. Sci. 500 (2002) 523-544. doi:10.1016/S0039-6028(01)01586-2.

[4] J. Erlebacher, M.J. Aziz, E. Chason, M.B. Sinclair, J.A. Floro, Spontaneous Pattern Formation on Ion Bombarded Si(001), Phys. Rev. Lett. 82 (1999) 2330-2333. doi:10.1103/PhysRevLett.82.2330.

[5] Facsko, Dekorsy, Koerdt, Trappe, Kurz, Vogt, Hartnagel, Formation of Ordered Nanoscale Semiconductor Dots by Ion Sputtering., Science. 285 (1999) 1551-1553. http://www.ncbi.nlm.nih.gov/pubmed/10477516 (accessed June 11, 2017).

[6] M. Teichmann, J. Lorbeer, B. Ziberi, F. Frost, B. Rauschenbach, Pattern formation on Ge by low energy ion beam erosion, New J. Phys. 15 (2013) 103029. doi:10.1088/1367-2630/15/10/103029.

[7] J.J. Vajo, R.E. Doty, E. Cirlin, Influence of $\mathrm{O}+2$ energy, flux, and fluence on the formation and growth of sputtering?induced ripple topography on silicon, J. Vac. Sci. Technol. A Vacuum, Surfaces, Film. 14 (1996) 2709-2720. doi:10.1116/1.580192.

[8] J. Shen, J. Kirschner, Tailoring magnetism in artificially structured materials: the new frontier, Surf. Sci. 500 (2002) 300-322. doi:10.1016/S0039-6028(01)01557-6.

[9] E. Doktorgrades, Mechanisms and Manipulation of Ion Beam Pattern Formation on Si ( 001 ) Sven Macko aus Duisburg, (2011). 